\title{
Escuela Digital: estrategias y materiales didácticos digitales en Educación
} Infantil y Primaria

\section{Escola Digital: estratégias e materiais didáticos digitais no Ensino Básico e Fundamental}

\section{Diana Marín ${ }^{1}\left[0\right.$; Pablo Joel Santana ${ }^{2}$ [D ; María Montserrat Castro ${ }^{3}$ [C] \\ ${ }^{1}$ Universitat de València, ${ }^{2}$ Universidad de La Laguna, ${ }^{3}$ Universidade da Coruña}

En la llamada de este monográfico afirmamos que "en los últimos tiempos, el impacto de la tecnología en todos los ámbitos de la vida cotidiana es innegable. La sociedad actual está expuesta a una gran revolución tecnológica, con cambios acelerados y nuevas exigencias formativas y laborales. El contexto educativo no es ajeno a dichos cambios y desde la educación se están haciendo esfuerzos, más o menos notables, dirigidos a responder a las diversas demandas sociales de la revolución tecnológica."

En este contexto de transformación tecnológica los y las docentes están implementando estrategias didácticas digitales que persiguen el cambio y la innovación en la escuela. Esta realidad se refleja también en los materiales didácticos digitales utilizados para el aprendizaje, que en muchas ocasiones son adaptados, o creados por el propio profesorado (Area 2017, 2020).

La conmoción que supuso el cierre de escuelas e institutos por varios meses a partir de febrero-marzo de 2020, por causa de la COVID-19, y la transición súbita a lo que se ha dado en llamar "enseñanza remota de emergencia" han acelerado esta transformación tecnológica que, aun con sus luces y sus sombras, hacen más valiosas las aportaciones de este monográfico (Hodges et al., 2020; Peirats, Rodríguez, San Martín, 2019). En este monográfico nos hemos centrado en las etapas de Educación Infantil y Primaria por las importantes diferencias que tienen respecto a otras etapas posteriores, por las características psicoe-
Na chamada deste volume, afirmamos que "nos últimos tempos, o impacto da tecnologia em todas as áreas da vida diária é inegável". A sociedade atual está exposta a uma grande revolução tecnológica, com mudanças aceleradas e novas exigências de formação e trabalho). 0 contexto educacional não é al heio a estas mudanças e na educação estão sendo feitos esforços, mais ou menos notáveis, para responder às diversas demandas sociais da revolução tecnológica".

Neste contexto de transformação tecnológica, professores e professoras estão implementando estratégias didáticas com recursos digitais que visam a mudança e a inovação na escola. Esta realidade também se reflete nos materiais didáticos digitais utilizados para a aprendizagem que, em muitos casos, são adaptados ou criados pelos próprios professores (Area 2017, 2020).

A comoção causada pelo fechamento de escolas e institutos durante vários meses, de fevereiro a março de 2020, devido à COVID-19, e a súbita transição, para o que foi chamado de "ensino remoto de emergência", aceleraram esta transformação tecnológica que, mesmo com suas luzes e sombras, torna mais valiosas as contribuições deste número monográfico (Hodges et al., 2020; Peirats, Rodríguez, San Martín, 2019).

Enfocamos as etapas de Educação Infantil e os anos iniciais do Ensino Fundamental (Básico), devido às importantes diferenças que têm em relação a outras etapas posteriores e às características psicológicas do desenvolvimento dos 
volutivas del alumnado de cada una de esas etapas, y porque consideramos de mucho interés ahondar en los resultados obtenidos en investigaciones recientes en este campo.

El monográfico reúne diez textos resultados de sendas investigaciones realizadas en distintos contextos, España, Portugal, Colombia, Venezuela y Brasil, escritos en diversos idiomas, elaborados por equipos de investigadores e investigadoras. Cuatro de los artículos tienen como primer firmante un hombre, pero la mayoría de las participantes son autoras. Se han seleccionado artículos surgidos de proyectos de investigación, pero también de experiencias específicas. Algunos de ellos se centran en algún tramo de educación preescolar, o Educación Infantil, y otros en algún tramo de educación elemental, o Educación Primaria.

Los primeros cuatro artículos son fruto de tres proyectos de investigación desarrollados en España. Los dos primeros son sendos estudios de caso comparativos -uno referido a cuatro centros de dos comunidades autónomas y el otro referido a tres centros de tres comunidades autónomas- en los que se estudian aulas de cuarto, quinto y sexto de primaria. Corresponden a dos proyectos de investigación diferentes. El primero de ellos se ocupa de los aprendizajes para la vida y muestra que, aunque las escuelas y sus agentes -profesorado, alumnado y familias- avanzan hacia la integración de las tecnologías, persisten carencias en cuestiones tan importantes como la igualdad, la diversidad o la participación. El segundo se ocupa de la creación de materiales didácticos digitales y su uso, y pone de manifiesto que los y las docentes abogan por la creación de sus propios materiales a fin de adaptarlos al alumnado al que atienden y por diseñar una propuesta de enseñanza contextualizada. El tercer y cuarto artículo surgen de un mismo proyecto de investigación en alunos e alunas. Também consideramos de grande interesse aprofundar os resultados obtidos em pesquisas recentes neste campo.

O volume reúne dez artigos, resultados de pesquisas realizadas em diferentes contextos, Espanha, Portugal, Colômbia, Venezuela e Brasil, escritos em diversos idiomas e elaborados por equipes de pesquisadores e pesquisadoras. Quatro artigos são assinados por homens, mas a maioria de autores participantes são mulheres. Os artigos foram selecionados a partir de projetos de pesquisa, mas também de experiências específicas. Alguns deles se focaliza a etapa da Educação Infantil, e outros os anos iniciais do Ensino Fundamental (Básico).

Os quatro primeiros são fruto de três projetos de pesquisa desenvolvidos na Espanha. Os dois primeiros são estudos de casos comparativos - um referente a quatro instituições de ensino de duas comunidades autônomas e o outro referente a três escolas de três comunidades autônomas - nas quais se analisam turmas do quarto, quinto e sexto ano do Ensino Fundamental. Correspondem a dois projetos de pesquisa diferentes. O primeiro versa sobre o aprendizado para a vida $e$ mostra que, embora as escolas e seus agentes - professores, alunos e famílias - estejam caminhando para a integração de tecnologias, ainda há carências em questões tão importantes como a igualdade, diversidade ou participação.

O segundo refere-se à criação de materiais didáticos digitais e seu uso, evidenciando que os professores defendem a criação de seus próprios materiais a fim de adaptálos para seus alunos e a elaboração de uma proposta de ensino contextualizada. O terceiro e quarto artigos surgem da mesma pesquisa, que está sendo desenvolvida, sobre a criação e uso de recursos digitais por alunos e alunas com idades entre 3 e 6 anos, em escolas e em suas casas. 0 terceiro artigo consiste na 
curso sobre la creación y uso de recursos digitales por alumnos y alumnas de entre 3 y 6 años en los centros escolares y en las familias. El tercer artículo consiste en un análisis de los materiales digitales de los repositorios institucionales de tres comunidades autónomas. Se concluye que los materiales más frecuentes son objetos digitales en lengua castellana, elaborados por docentes de modo individual, sin actualizaciones recientes, mayormente de conocimiento del entorno y lenguaje, pero que no especifican la edad concreta a la que van dirigidos. El cuarto artículo analiza las características de las 23 apps más populares durante junio de 2020. Se encontró que las apps contenían elementos curriculares, aunque su fin era el entretenimiento. Sin embargo, no se basan en el aprendizaje activo, son poco accesibles, presentan contravalores y prima la publicidad intrusiva.

El quinto y sexto artículos abordan el desarrollo del pensamiento crítico. El primero, un estudio de caso, recoge los resultados de un proyecto a gran escala en Portugal que tenía como objetivo integrar las diversas áreas del currículo y el aprendizaje específico del pensamiento crítico en el jardín de infancia. Fue implementado mediante una plataforma digital y cinco propuestas. Se analiza el impacto de una de ellas y se concluye que el uso de recursos didácticos digitales permitió ejercitar la imaginación y la creatividad, promover la curiosidad por el conocimiento del medio, y desarrollar las capacidades de toma de decisiones e interpretación de un cortometraje, entre otras. El sexto artículo presenta una investigación-acción para la mejora de la lectura crítica con un grupo de estudiantes de primaria de Colombia. Se observó una mejora en el desempeño de la lectura crítica, pero también el impacto positivo del uso de la tecnología en el proceso de enseñanza y aprendizaje en análise dos materiais digitais dos repositórios institucionais de três comunidades autônomas. Conclui-se que os materiais mais frequentes são recursos digitais em espanhol, preparados por docentes individualmente, sem atualizações recentes, em sua maioria sobre conhecimento do ambiente e língua, mas que não especificam a idade concreta do público-alvo. O quarto artigo analisa as características dos 23 aplicativos mais populares baixados durante junho de 2020. Constatouse que os apps continham elementos curriculares, embora seu objetivo fosse o entretenimento. Entretanto, não se baseiam numa aprendizagem ativa, não são muito acessíveis, têm contravalores e predomina a publicidade intrusiva.

0 quinto e sexto artigos abordam o desenvolvimento do pensamento crítico. O primeiro, um estudo de caso, reúne os resultados de um projeto de grande escala em Portugal, que visava integrar as diversas áreas do currículo e a aprendizagem específica do pensamento crítico no jardim de infância. Foi implementado através de uma plataforma digital e cinco propostas. Foi feita uma análise sobre o impacto de uma delas, chegando à conclusão de que o uso de recursos didáticos digitais permite exercitar a imaginação e a criatividade, promover a curiosidade pelo conhecimento do meio e desenvolver a capacidade de tomar decisões e de interpretar um curta-metragem, entre outras. O sexto artigo apresenta uma pesquisa-ação para melhorar a leitura crítica com um grupo de estudantes dos anos iniciais do ensino fundamental na Colômbia, demonstrando uma melhoria no desempenho de leitura crítica, mas também o impacto positivo do uso da tecnologia no processo de ensino-aprendizagem, na motivação dos alunos e alunas, e na melhoria do próprio processo de ensino-aprendizagem. 
la motivación de los y las estudiantes y en la mejora del propio proceso de enseñanza y aprendizaje.

El séptimo artículo informa de una investigación cuyo objetivo era elaborar una propuesta pedagógica de uso de pequeños cortometrajes para aprender sobre el medio ambiente en educación preescolar (3-6 años). Se desarrolló en Venezuela con un grupo de 38 docentes. Se concluyó que el material tecnológico elaborado ( 12 cortos) es pertinente para la etapa preescolar y que la combinación mensaje-sonido-imagen permite potenciar el desarrollo intelectual de los niños y alcanzar en breve tiempo los aprendizajes que se pretende lograr. El octavo artículo, como el anterior, va dirigido a alumnado en situación de vulnerabilidad social, pero se ocupa del aprendizaje del pensamiento computacional mediante un proceso de investigación-acción desarrollado en Colombia con niños y niñas de entre 9 y 13 años. El análisis de la información recogida permitió identificar diversos aprendizajes. Las niñas y los niños adquieren aprendizajes espontáneamente, más allá de lo que establecen sus maestras y maestros, son capaces de identificar sus propios aprendizajes y perciben con claridad la utilidad de las tecnologías. Tienen sentimientos encontrados respecto a las tecnologías, y viven de modo singular la pertenencia a grupos de interés en línea y la censura de contenidos. También se perciben como agentes que pueden enseñar a sus padres. En la última parte de este artículo se reflexiona sobre las implicaciones de estos aprendizajes.

El noveno artículo persigue evaluar la contribución del uso de juegos digitales al desarrollo de la conciencia fonológica de niños y niñas con dificultades de aprendizaje en el proceso de alfabetización. Participaron 14 estudiantes de tercero y cuarto de primaria de dos escuelas en Brasil. Tras un período de intervención
O sétimo artigo apresenta uma pesquisa cujo objetivo era elaborar uma proposta pedagógica para o uso de curtas-metragens para aprender sobre o meio ambiente na Educação Infantil (3-6 anos). Desenvolvida na Venezuela com um grupo de 38 professores, concluiu que o material tecnológico produzido (12 microvídeos) é relevante para a etapa pré-escolar e que a combinação de mensagem-som-imagem permite melhorar o desenvolvimento intelectual das crianças e alcançar em pouco tempo o aprendizado desejado. $O$ oitavo artigo, como o anterior, foca estudantes em situações de vulnerabilidade social, mas aborda a aprendizagem do pensamento computacional através de um processo de pesquisa-ação desenvolvido na Colômbia, com crianças com idades entre 9 e 13 anos. A análise das informações coletadas permitiu identificar diversos aprendizados. As crianças aprendem espontaneamente, além do que é estabelecido por seus professores, são capazes de identificar seu próprio aprendizado e de perceber claramente a utilidade das tecnologias. Têm sentimentos contraditórios sobre as tecnologias e experimentam de uma forma única o pertencimento a grupos de interesse on-line e a censura de conteúdo. Também se veem como agentes que podem ensinar seus pais. Na última parte deste artigo faz-se uma reflexão sobre as implicações dos aprendizados. O nono artigo avalia a contribuição do uso de jogos digitais para o desenvolvimento da consciência fonológica de crianças com dificuldades de aprendizagem no processo de alfabetização. Participaram do estudo catorze estudantes do terceiro e quarto ano do Ensino Fundamental de duas escolas brasileiras. Após um período de intervenção de dois meses, foi confirmado que o uso de jogos digitais significou uma experiência positiva no processo de alfabetização no contexto escolar. 
de dos meses, se corroboró que el uso de juegos digitales significó una experiencia positiva en el proceso de alfabetización en el contexto escolar.

El último artículo consiste en el análisis de las potencialidades pedagógicascurriculares de 16 planes semanales de aprendizaje a distancia del primer ciclo de primaria de dos editoriales portuguesas. El análisis de los recursos sugiere que no se trabaja desde un enfoque de currículo integrado, que la actitud esperada de alumnas y alumnos es más bien pasiva y que se da poca importancia a la evaluación. Sin embargo, en una situación prácticamente sin precedentes, son contraejemplos de otra solución tecnológica relevante para esa etapa formativa.
O último artigo consiste na análise das potencialidades pedagógicas de 16 planos de trabalho semanais de ensino à distância do primeiro ciclo do Ensino Básico de duas editoras portuguesas. A análise dos recursos sugere que não são trabalhados a partir de uma abordagem curricular integrada, que a atitude esperada dos alunos e alunas é bastante passiva e que se dá pouca importância à avaliação. No entanto, numa situação praticamente sem precedentes, são contraexemplos de outra solução tecnológica relevante para essa etapa de formação.

\section{Referencias / Referências}

Area, M. (2017). La metamorfosis digital del material didáctico tras el paréntesis Gutenberg. Revista Latinoamericana de Tecnología Educativa. RELATEC, 16(2), 13-28.

Area, M. (coord.). (2020). Escuel@Digit@I. Los materiales didácticosen la Red. Barcelona: Graó.

Hodges, Ch., Moore, S., Lockee, B., Trust, T. y Bond, A. (2020, March 27). The Difference Between Emergency Remote Teaching and Online Learning. EducauseReview. https://bit.ly/3h6Bjh5

Peirats, J., Rodríguez, J., \& San Martín, Á. (2019). Controversias del liderazgo escolar en la implantación de materiales digitales. Campus Virtuales, 8(2), 19-34. 